\section{Carbohydrate Antigen 242}

S. Holdenrieder ${ }^{1}$ und P. Stieber ${ }^{2}$

${ }^{1}$ Institut für Laboratoriumsmedizin, Deutsches Herzzentrum München, Klinik an der Technischen Universität München, München, Deutschland

${ }^{2}$ Institut für Laboratoriumsmedizin, Klinikum der Universität München (LMU), München, Deutschland

\section{Synonym(e) CA 242}

Englischer Begriff carbohydrate antigen 242

Definition Carbohydrate antigen 242 wurde durch den monoklonalen Antikörper C 242 ( $\triangleright$ Antikörper) definiert, der ein Epitop eines polymorphen epithelialen Muzins erkennt.

Synthese - Verteilung - Abbau - Elimination Physiologisch kommt CA 242 in geringen Mengen in Geweben des Gastrointestinaltrakts vor.

Untersuchungsmaterial - Entnahmebedingungen Serum, Aszites, Pleurapunktat.

Analytik $>$ Immunradiometrischer Assay (IRMA).

\section{Konventionelle Einheit kU/L.}

Referenzbereich - Erwachsene $<20 \mathrm{kU} / \mathrm{L}$ (methodenabhängig).

Indikation Evtl. Therapiekontrolle und Nachsorge beim Pankreaskarzinom.

Interpretation CA 242 kann zur Therapiekontrolle und Nachsorge des Pankreaskarzinoms eingesetzt werden. Allerdings bietet CA 242 keine Vorteile gegenüber dem CA 19-9 und sollte wegen nicht vorhandener Komplementarität zu CA 19-9 mit diesem nicht kombiniert werden.

In den aktuellen Richtlinien der European Group on Tumor Markers (EGTM) zum Gebrauch von Tumormarkern beim Pankreaskarzinom wird CA 19-9 im Vergleich zu CA 242 der Vorzug gegeben. Aufgrund der fehlenden Automatisierung von CA-242-Testen ist dieser Marker eher als historisch zu betrachten.

Diagnostische Wertigkeit Evtl. Pankreaskarzinom: Therapiemonitoring, Rezidiverkennung.

\section{Literatur}

Duffy MJ, Sturgeon C, Lamerz R et al (2010) Tumor markers in pancreatic cancer: a European Group on Tumor Markers (EGTM) status report. Ann Oncol 21:441-447

Lamerz R, Dati F, Feller AC et al (1998) Tumordiagnostik: Tumormarker bei malignen Erkrankungen. Behring Diagnostika, München 